\title{
Neuroimmunological Aberrations and Cerebral Asymmetry Abnormalities in Schizophrenia: Select Perspectives on Pathogenesis
}

\author{
Ganesan Venkatasubramanian ${ }^{1,2}$, Monojit Debnath ${ }^{3}$ \\ ${ }^{1}$ The Schizophrenia Clinic, Department of Psychiatry, National Institute of Mental Health and Neurosciences, ${ }^{2}$ Translational Psychiatry \\ Laboratory, Neurobiology Research Centre, National Institute of Mental Health and Neurosciences, ${ }^{3}$ Department of Human Genetics, \\ National Institute of Mental Health and Neurosciences, Bangalore, India
}

Within the wide-ranging gamut of factors that comprise gene-environment interactions postulated to underlie schizophrenia, the crosstalk between environmental factors and feto-maternal immune components has been put forth as one of the important mechanisms that increase the risk towards schizophrenia in the offspring. Interestingly, immune factors have been shown to critically modulate the brain development during the prenatal stages. Moreover the past many decades, influential theoretical propositions and evidence base (albeit not unequivocally) have compellingly linked prenatal sex hormonal status to critically provoke long lasting immunological changes and subsequently affect developmental programming of cerebral asymmetry in schizophrenia. In this review, we summarize the select perspectives emphasizing the role of neuroimmunoendocrine pathways in anomalous cerebral asymmetry in contemporary understanding of schizophrenia pathogenesis.

KEY WORDS: Schizophrenia; Immune abnormalities; Cerebral asymmetry; Estrogens; Neurodevelopment.

\section{INTRODUCTION}

Schizophrenia is a complex neuropsychiatric disorder that is characterized by delusions, hallucinations, disorganized behavior and progressive cognitive deficits with postulated neurodevelopmental origin. ${ }^{1,2)}$ The neurodevelopmental pathogenetic model implicates schizophrenia as a behavioural outcome of impaired neurodevelopmental processes that begins long before the onset of clinical symptoms and is caused by a combination of genetic, immune and environmental factors. ${ }^{3)}$ Among these factors, multiple susceptibility genes regulated by hypoxia are shown to enhance the risk of schizophrenia by interacting with serious obstetric complications. ${ }^{4)}$ In addition, it is noteworthy that prenatal infections with resultant persistent pro-inflammatory state leading to aberrant neurodevelopment as well as obstetric complications are also shown to confer increased risk for schizophrenia. ${ }^{5)}$ Epidemiological observations strongly support the associ-

Received: December 9, 2013 / Accepted: February 3, 2014 Address for correspondence: Ganesan Venkatasubramanian, $\mathrm{MD}, \mathrm{PhD}$

Department of Psychiatry, National Institute of Mental Health and Neurosciences (NIMHANS), Bangalore 560029, India

Tel: +91-80-26995256, Fax: +91-80-26564830

E-mail: venkat.nimhans@yahoo.com ation between elevated risk for schizophrenia in the offspring and prenatal exposure to influenza, toxoplasma, rubella, genital-reproductive infections and various other infections. ${ }^{6)}$ It is noteworthy that multiple immune mediators such as cytokines, major histocompatibility complex (MHC) molecules etc. critically modulate the brain development during the prenatal stages. ${ }^{7,8)}$ However, prenatal infection by various viral, bacterial and protozoan pathogens have been found to alter the expression of various immune molecules and contribute to early development etiology of schizophrenia. Interestingly, over the past many decades, influential theoretical propositions (GeschwindBehan-Galaburda hypothesis ${ }^{9-11)}$ being principal among them) and evidence base (albeit not unequivocally) have compellingly linked prenatal sex hormonal status to altered immune functions. ${ }^{12)}$ Collectively, such sex hormones and immune system interactions during prenatal period have been shown to modulate cerebral asymmetry (comprehensively reviewed in the article of Stoyanov et $a l{ }^{13)}$ ). Accumulating evidence increasingly implicates the significance of immune-inflammatory and neuroendocrinological processes as well as abnormalities in cerebral asymmetry in the pathogenesis of at least in a subset of patients with schizophrenia. In this review, we summarize the select perspectives emphasizing the role of im-

(c) This is an Open-Access article distributed under the terms of the Creative Commons Attribution Non-Commercial License (http://creativecommons.org/licenses/by-nc/3.0) which permits unrestricted non-commercial use, distribution, and reproduction in any medium, provided the original work is properly cited. 
mune-inflammatory aberrations and their interactions with cerebral asymmetry abnormalities in contemporary understanding of schizophrenia pathogenesis.

\section{MAIN DISCOURSE}

\section{Immune Abnormalities in Schizophrenia: A Select Overview}

Fetal brain development is particularly vulnerable to environmental insults, and deviations from the normal course of brain development are assumed to underlie several mental disorders, including schizophrenia. Epidemiological studies have indicated that maternal infections during pregnancy increase the risk for these disorders in the offspring. Multiple lines of evidence from epidemiologic, clinical, and preclinical studies have provided evidence that gestational exposure to infection contributes to the etiology of schizophrenia. ${ }^{5)}$ Importantly, season of birth findings in schizophrenia robustly implicate birth in winter or spring months as a significant risk factor for schizophrenia; more specifically, a wealth of studies suggest that the prevalence of influenza in winter months might contribute significantly to this risk. ${ }^{14)}$ This also implies the influence of season of birth on the immune-mediated processes in schizophrenia. It is now widely appreciated that, the maternal cytokine response to infections may play a crucial role in this association, because the induction of cytokines is a fundamental immunological event triggered by virtually any infection. ${ }^{15,16)}$ Studies on schizophrenia incidence after epidemics of influenza offer unequivocal support to increased incidence of this disorder among exposed offspring. Examination of medical records of more than 12,000 pregnant women revealed threefold to sevenfold increase in the risk for schizophrenia in the offspring following second-trimester respiratory infection in the mother. ${ }^{17)}$ Given the high prevalence of influenza infection, it has been estimated that about $14-21 \%$ of schizophrenia cases could have been due to maternal infection. ${ }^{15)}$

Interestingly, it is the maternal immune response, rather than direct infection of the foetus which has been described to be responsible for the increased incidence of schizophrenia in the offspring of mothers who suffer infections during pregnancy. ${ }^{15)}$ Indeed, animal models of maternal immune activation (MIA) have revealed intriguing findings indicating behavioral, neurochemical, and neurophysiologic abnormalities consistent with observations in schizophrenia. ${ }^{5)}$ MIA is also linked to the well-established dopamine hypothesis of schizophre- nia. ${ }^{18)}$

Moreover, several studies have shown that after MIA cytokine levels are altered in the maternal serum as well as the amniotic fluid, placenta, and fetal brain ${ }^{19-21)}$; hence, it has been argued that cytokines, by virtue being the major factors in influencing innate immune response, are the logical candidates for disruption of fetal brain development. ${ }^{16)}$ Among various cytokines, rigorous evidence based on animal model research has demonstrated that elimination of interleukin (IL)-6 in the MIA model almost completely abolishes abnormal behaviors and transcriptome changes in the offspring suggests that searches for other mediators of MIA should be directed upstream and downstream of IL-6, rather than in other signaling pathways. ${ }^{1622-25)}$ In addition, the following robust observations further strengthen the postulated role of IL- 6 in schizophrenia pathogenesis: 1) Rigorous meta-analytic studies supporting significantly higher serum levels of IL-6 in schizophrenia patients that correlate with symptom severity ${ }^{26)}$; 2) Association of schizophrenia with IL-6 gene polymorphism ${ }^{27}$; 3) Potential influence on hippocampus by serum IL- 6 with hippocampus being the most important brain regions implicated in schizophrenia $\left.{ }^{28} ; 4\right)$ IL-6 playing a vital role in established models like 'ketamine model' of schizophrenia ${ }^{29)}$; 5) IL-6 being implicated in foetal pathogenetic model of neurodevelopmental aberrations in schizophrenia. ${ }^{16)}$

The signaling of IL- 6 , a pleiotropic cytokine, occurs through heterodimerization of gp 130 and IL- 6 receptor on the cell surface. ${ }^{30)}$ With relevance to the specific mechanism through which IL-6 enhances the risk for schizophrenia in the MIA model, three major sites of signaling have been suggested namely - 1) the maternal circulation, 2) the maternal/fetal interface (i.e., the placenta), and 3) the fetal brain. ${ }^{16}$

The impact of IL- 6 on the maternal immune system can result in activation of lymphocyte migration and cytotoxicity and degrading maternal tolerance of the fetus. During normal pregnancy, a shift in basal cytokine production and other immune system related modulations occur to prevent rejection of the fetus. ${ }^{31,32)}$ However, in the context of MIA, an increased level of IL-6 leads to enhanced production of certain maternal antibodies that could potentially cross-react with the fetal brain with resultant damage. ${ }^{33)}$ The adverse impact of IL-6 on the maternal-fetal interface (placenta) could alter the transfer of nutrients, hormones, or other key molecules to the fetus. This could have significant effects on transfer of potentially harmful proteins (i.e., antibodies) into the fetal environment, or 
could allow maternal immune cells to infiltrate the fetus. $^{16)}$

With relevance to the direct impact of IL- 6 on fetal brain, it is important to note that IL-6 is central to inflammation-induced working memory disruption. ${ }^{34)}$ Also, IL-6 can regulate brain-derived neurotrophic factor (BDNF) expression, ${ }^{35)}$ increased levels of IL-6 can lead to decrease in BDNF in embryos and placentas. The compelling role for BDNF in the pathogenesis of schizophrenia is supported by various lines of evidence (see the articles of Buckley et al $^{36)}$ and Favalli et $a l^{37)}$ for reviews). BDNF, the most widely distributed neurotrophin in the central nervous system, is highly expressed in brain regions that are critically implicated in the pathogenesis of schizophrenia - the prefrontal cortex and hippocampus. ${ }^{38)}$ BDNF has been demonstrated to interact with various neurotransmitter systems that are implicated in schizophrenia, such as dopamine, glutamate, serotonin and GABA. $^{39)}$

To generalize this further, it has been suggested that activation of pro-inflammatory cytokines (with robust support for IL- ${ }^{15,16)}$ ), mediate the neurodevelopmental effects of maternal infections on the offspring. While these cytokines can modulate neuronal differentiation, survival, dendrite growth and complexity, they have also been critically involved in the precipitation of the long-term behavioral, cognitive and pharmacological consequences of prenatal immune challenge. ${ }^{21,40)}$

Apart from BDNF, the deficiency of insulin-like growth factor-1 (IGF-1) - another critical neurotrophic factor which is essential for optimal neurodevelopment has been demonstrated in antipsychotic-naïve schizophrenia. ${ }^{41)}$ IGF-1 has neuroprotective and antiapoptotic properties that are crucial during neurodevelopment. ${ }^{42)}$ Possibly low IGF-1 levels might render the brain more vulnerable to neurodevelopmental insults potentially culminating in schizophrenia. Interestingly, IL-6 inhibits the secretion of IGF-I and its biological activity. ${ }^{43)}$ Cerebral damage in foetal pro-inflammatory states has been reported to be associated with high IL-6 and low IGF-1 levels. These studies support significant interaction between IL-6 and IGF-1. Increased IL- $6^{26)}$ as well as deficient IGF- ${ }^{41)}$ has been demonstrated in schizophrenia. ${ }^{44)}$

Deficits in hippocampus with associated disturbances in its network brain regions are among the most established findings in schizophrenia. ${ }^{45-47)}$ Recent studies suggest compelling support for the effect of IL- $6^{28)}$ and IGF-1 ${ }^{48)}$ on hippocampal structure and function. Peripheral levels of IL-6 have been shown to have a significant in- verse correlation with gray matter volume of hippocampus. ${ }^{28)}$ Both IGF-1 and IL-6 influence the functioning of hippocampus - an important brain region involved the pathogenesis of schizophrenia, IGF-1 improves hippocampal functioning ${ }^{42)}$ whereas IL-6 damages it. The human IL-6 gene is located on chromosome 7p15-7p21 and IL-6 expression is primarily regulated by alterations in gene transcription. A functional $\mathrm{G} \rightarrow \mathrm{C}$ single-nucleotide polymorphism at position -174 of the promoter has been described. ${ }^{49)}$ Genetic and expression studies suggested that the GG allele was associated with greater induction of IL- 6 compared with the GC or CC alleles. Hence, it is possible that MIA might result in persistent over-activation of glial cells which could be more pronounced in individuals who are predisposed towards greater induction of IL-6 and various other inflammatory cytokines; these pathogenetic mechanisms might lead to hippocampal network damage culminating in symptoms of schizophrenia.

\section{Cerebral Asymmetry Abnormalities in Schizophrenia}

The concept of asymmetry being the defining feature of human brain was emphasized by the assertion of Paul Broca $^{50)}$ that: "Man is, of all the animals, the one whose brain is most asymmetrical. He is also the one who possesses most acquired faculties. Among these faculties $\cdots$ the faculty of language holds pride of place. It is this that distinguishes us most clearly from the animals" (see the article of $\mathrm{Crow}^{51)}$ for review).

In earlier times, brain asymmetry was considered to be specific of the human species. However, functional and structural asymmetries have since been described in other mammals and birds ${ }^{52,53)}$; nonetheless, among mammals, and even within the primate lineage, humans frequently possess the most asymmetrical brain. ${ }^{54)}$ In the human brain, the principal asymmetries are described as a torque pushing the right frontal area forward and the left occipital region backward (petalias) - called as the "Yakovlevian torque"; along with this torque, the right Sylvian fissure is relatively raised and shortened in comparison with the left Sylvian fissure and this creates a striking difference in the shapes of the left and right posterior temporal regions and a larger left planum temporale (as summarized in the article of Glasel et $a l .{ }^{54)}$ ). Since this right-left torsion affects the posterior temporal region, which is involved in elaborate auditory computations among which phonetic representations on the left side, the relation between this macro-structural feature and the emergence of language is an area of strong research interest. Also, it has been demonstrated that the white matter volume of the underlying 
Heschl's gyri is larger on the left than on the right side. Moreover, at the microscopic level, left auditory cortex has been shown to have bigger pyramidal cells with thicker myelinated fibers (as summarized in the article of Glasel et $a{ }^{54)}{ }^{54)}$.

At the time of embryonic development, the cells of the brain migrate toward the surface, forming minicolumns in the cortex. These are grouped into larger macrocolumns to form the basis of the mapping of functions across the brain's surface. As per the radial unit hypothesis, proliferation of radial minicolumnar units of cells underlies the expansion of different cortical surface regions during development and across species. While many of the brain asymmetries demonstrated in humans are also found in one or other non-human primate, the difference in minicolumn width in humans between left and right planum temporale has not been reported in chimpanzees. ${ }^{55}$

Auditory cortex in the superior temporal gyrus develops a clear columnar cell distribution by the third trimester of fetal life, which is established in early childhood, although axonal maturation continues up to at least 12 years of age. ${ }^{56)}$ The functional macrocolumnar organization in primary auditory cortex with topological mapping of stimulus features such as frequency and ear preference can be inferred from the radial organization of physiological response properties. Asymmetry in columnar organization facilitates these asymmetries in that the macrocolumns are more widely spaced in the left than in the right auditory association cortex. ${ }^{57)}$

In this context, it is important to note that the superior temporal gyrus, which contains the auditory cortex, including the planum temporale, is the most consistently altered neocortical structure in schizophrenia. ${ }^{58)}$ In a post-mortem brain study, ${ }^{59)}$ it was observed that normal age-associated changes in planum temporale were not found in schizophrenia. Minicolumn distribution in planum temporale and Heschl's gyrus (as assessed on Nissl-stained sections by semi-automated microscope image analysis) revealed that the cortical surface area of planum temporale in the left hemisphere was positively correlated with its constituent minicolumn spacing in patients and controls. In schizophrenia patients, surface area asymmetry of planum temporale was decreased with this asymmetry abnormality being resultant of reduction in the left hemisphere. The relationship between cortical asymmetry and the connecting, inter-hemispheric callosal white matter was also investigated; minicolumn asymmetry of both Heschl's gyrus and planum temporale was correlated with axon number in the incorrect sub-regions of the cor- pus callosum in patients. Also, the spacing of minicolumns was altered in a sex-dependent manner due to the absence of age-related minicolumn thinning in schizophrenia suggesting a failure of adult neuroplasticity that maintains neuropil space. This was inferred to indicate the arrested capacity to absorb anomalous events and cognitive demands to result in vulnerability to develop schizophrenia. $^{59)}$

In summary, one of the uniquely human asymmetry namely, the minicolumn asymmetry in the planum temporale $^{55)}$ - has been found to be aberrant in schizophrenia. ${ }^{59)}$ Asymmetry abnormalities indicative of disturbed cerebral lateralization in schizophrenia patients with a significant leftward shift in the handedness has been established in various comprehensive reviews. ${ }^{60-62)}$ Moreover, a number of anatomical postmortem and functional imaging studies have shown that schizophrenia is characterized by reduced cerebral asymmetry, ${ }^{51,63,64)}$ most likely resulting from abnormalities involving gene-environmental interactions responsible for cerebral lateralization aberration $^{65)}$ that has been considered as central to the pathogenesis of schizophrenia.

\section{Linking Immune Factors with Cerebral Asymmetry}

The Geschwind-Behan-Galaburda (GBG) hypothesis links the cerebral lateralization with prenatal testosterone exposure and explains differential immune profile. ${ }^{9-11,66)}$ It proposes that testosterone levels in the fetal milieu dictate the development of the left cerebral hemisphere. Elevated levels of testosterone retard the development of the left hemisphere causing "abnormal dominance" and leading to modified cerebral lateralization, disrupted early language development, immune dysfunction and predilection for various other disorders. Thus, the GBG hypothesis attempts to explain the normal distribution and the inter-individual differences in various domains of cognitive functioning in humans as a function of prenatal sex hormonal environment. ${ }^{9-11,66)}$

The postulated mechanistic basis for the hypothetical link between cerebral dominance and immune aberrations as stated by Geschwind and Behan ${ }^{67)}$ is as follows: "During fetal life the immune system is also maturing. Testosterone has important suppressive effects on the thymus both in utero and after birth. Thus, during periods of increased testosterone effects on left brain development, maturation of the immune system is also likely to be affected. There are studies that support the hypothesis that the fetal thymus controls development of lymphocytes which are responsible for recognition of self-antigens and 
thus for prevention of autoimmunity. Suppression of thymic growth during fetal life might therefore favor the development of autoimmunity in later life."

Following this seminal report, a number of studies supporting the GBG model have identified a relationship between left-handedness, anomalous language or visuospatial brain dominance and immune diseases. ${ }^{68-76)}$ While GBG hypothesis was commented as a potential 'large' theory (from the perspectives of Popper's 'small' versus 'large' theories) that not only accounted for its original data but extending that theorizing to such an extent that, if true, it would require a radical rethinking of many areas of biology and medicine, however "the very size and complexity of the Geschwind theory however means, that it is not open to simple 'falsification' in Popper's sense" and it was also perceived that "this theory has a concentric series of defensive hypotheses around it, which can be falsified without the central theory being effectively challenged." ${ }^{\text {,77) }}$

Summarizing a number of studies that did not support the GBG hypothesis, ${ }^{78)}$ the mutually incompatible observations (from the viewpoint of Geschwind theory) that on one hand boys are more likely to be diagnosed as reading-disabled than girls, and on the other hand, men do not have higher rates of most immune-mediated disorders than women was considered as strongly negating the important claim of the GBG hypothesis. Also, the finding that certain autoimmune diseases like systematic lupus erythematosus, multiple sclerosis, myasthenia gravis, thyroid disease, arthritis etc. ${ }^{79)}$ are more prevalent in women than men as well as the implication of estrogen rather than testosterone to underlie autoimmune pathogenesis ${ }^{80)}$ further argued against the claim by Geschwind and co-workers.

Motivated by certain additional reports that were supportive of GBG theory (for example, the observation that two sides of the brain have been demonstrated to be involved in different ways in the modulation of immune responses ${ }^{81,82)}$ the existence of functional asymmetry of the immune system and the role of the brain hemispheres, differential postoperative changes in $\mathrm{T}$ cell indices in epilepsy patients as dependent upon the resection involving dominant or non-dominant hemisphere - as summarized by Legen $e t a l .{ }^{83)}$ ), the results of a recent study expanded further the gamut of relationship between the development of non-right-handedness, the anomalous cerebral dominance and the immune system beyond the frequency of immune diseases and that of humoral immunity into the boundaries of cellular immunity also.
In this study, when compared with the right handed individuals, the left handed ones demonstrated a significant decrease in the inflammatory cell types involving total $\mathrm{T}$ cells, T-helper cells, HLA-DR (HLA-DR is a MHC class II cell surface receptor encoded by the human leukocyte antigen [HLA] complex on chromosome 6 region 6p21.31) and natural killer cells. The authors of this study speculated a mediating role by dopamine and based on their HLA findings they have indicated that it might be worthwhile to investigate in more detail whether the MHC, which can be found on both astrocytes and lymphocytes, plays any role as a link between neuronal and immunological development. ${ }^{83)}$ Interestingly, it has been demonstrated that in nestling gull chicks, embryonic exposure to elevated levels of androgens negatively influenced both cell-mediated and humoral immunity ${ }^{84)}$ In summary, recent interest on immune factors' influences on brain lateralization have provided compelling evidence towards a robust link between them; however, this area needs to be clarified further with comprehensive and systematic studies to unravel the complexities of these interactions.

\section{Neuroimmunology, Brain Asymmetry and Pathogenesis of Schizophrenia}

While the studies summarized in the previous section supports a compelling link between the immune factors and cerebral lateralization processes, various lines of evidence as mentioned below suggest the relevance of this relationship in schizophrenia pathogenesis. For instance, MHC related brain deficits have been shown to be linked with microglia abnormalities that are considered as critical with respect to the pathogenesis of schizophrenia (as summarized in the article of Debnath et al. ${ }^{85}$ ). In a postmortem brain study examining the frontal and temporal cortices of schizophrenia patients, it was observed that degeneration of microgial cells with a stronger expression of MHC-II might have led to the exacerbation of structural damage and psychotic symptoms. ${ }^{86)}$ This was postulated to result most likely from the primary impairment of the neuron-glia communication that damages their immunocompetent function. ${ }^{86)}$ Moreover, aberrant hemispheric lateralization involving HLA Class-II expressing microglia was reported to be associated with schizophrenia. ${ }^{87}$

Neurodevelopmental insults elicited by pre-/peri-natal infections have consistently been shown to be associated with glial cell activation. ${ }^{88)}$ The central role of glial cells in the pathogenesis of various brain disorders has been highlighted by comprehensive literature that describe glia as 
critical for every major aspect of brain development, function and disease ${ }^{89)}$; it is noteworthy that brain pathology has been mentioned to a very great extent as a pathology of glia. ${ }^{90)}$ Interestingly, Kraepelin - an influential researcher in schizophrenia, had suggested a relationship between infection and later psychosis in the late 19th century and cited "changes in glia" as one of the primary anatomical changes within the brain. Once activated, glia produce a plethora of pro-inflammatory molecules including cytokines, chemokines etc. which might lead to the neuropathological changes in schizophrenia (see the article of Bilbo and Schwarz ${ }^{91)}$ for review). In a study that examined the evidence for microglial activation in schizophrenia, it was demonstrated that the density of HLA-DR-positive ameboid and ramified microglial cells was highest in the dorsolateral prefrontal cortex. More importantly, it was observed that there was a significantly decreased cerebral lateralization in schizophrenia also with regard to HLADR expression. ${ }^{87)}$

Though the microglia comprise about less than onetenth of the total brain cells, they react swiftly to even minor pathological changes in the brain and may directly lead to the neuronal degeneration by producing various pro-inflammatory cytokines and free radicals. ${ }^{92)}$ Comprehensive analyses support critical role for cytokines in schizophrenia pathogenesis. ${ }^{93)}$ Moreover, several postmortem brain studies on schizophrenia have indicated that the neuropathology of schizophrenia to be closely associated with microglial activation. Positron emission tomography studies have shown that microglia-mediated focal neuroinflammation involving hippocampus may play an important role in schizophrenia during psychosis. ${ }^{94)}$ In addition, it has been demonstrated that the antipsychotic medications, the mainstay for treating schizophrenia patients, have inhibitory effects on the release of inflammatory cytokines and free radicals from activated microglia, both of which have recently been known to cause a decrease in neurogenesis as well as white matter abnormalities in the brains of patients with schizophrenia. In fact, it has been argued that this microglia hypothesis of schizophrenia might shed new light on the therapeutic strategy for schizophrenia. ${ }^{92)}$ Interestingly, microglia based approach has been proposed to argue for novel therapeutic options like the utility of minocycline in the treatment of schizophrenia. ${ }^{95-97)}$ Clinical studies have indicated significant benefits of minocycline in treating schizophrenia patients. ${ }^{98-100)}$

Another critical line of evidence supporting immune aberrations to influence symptom genesis in schizo- phrenia is from the literature that have conclusively demonstrated that prenatal immune challenges result in persistent sensory gating deficits. ${ }^{101,102)}$ Sensory Gating Deficit hypothesis postulates that individuals with schizophrenia cannot inhibit or "gate" irrelevant sensory input, leading to sensory inundation and an overload of information reaching consciousness - leading to hallucinations and delusions. ${ }^{103,104)}$ The sensory gating problem observed in schizophrenia may result from neuronal hyperexcitability stemming from a defect in sub-cortical and cortical neuronal inhibitory pathways - especially involving "frontohippocampal network through thalamus". ${ }^{105)}$ The abnormal auditory gating observed in schizophrenia has been suggested to be a "fixed trait" that is genetically associated and that shows potential as a candidate endophenotype; reduced sensory gating ratio is one of the well replicated finding that has been documented in large percentage of schizophrenia patients. ${ }^{106,107)}$

Hippocampal network (inclusive of retrosplenial cortex) is one of the key neural generator of sensory gating ${ }^{105)}$; this is in tune with the robust evidence for hippocampal abnormalities in schizophrenia. ${ }^{45-47)}$ Interestingly, immune abnormalities involving elevated IL-6 and IL-2 have been demonstrated to underlie the sensory gating deficits $^{102)}$; importantly, these immune aberrations might potentially affect hippocampus. Recent studies suggest compelling support for the effect of IL- $6^{28)}$ on hippocampal structure and function. Peripheral levels of IL-6 have been shown to correlate with gray matter volume of hippocampus. ${ }^{28)}$ More importantly, asymmetry abnormalities involving hippocampus has been demonstrated to underlie schizophrenia pathogenesis. ${ }^{108,109)}$

One important neuroimmunoendocrine modulator that influences all these factors described above (namely cytokines, hippocampal function, sensory gating and schizophrenia) is estrogen. Estrogen is an important immunomodulator $^{110,111)}$; more specifically, selective estrogen receptor modulators have been shown to decrease the IL-6 gene expression. ${ }^{112}$ Also, estrogen has neuroprotective effects on hippocampus. ${ }^{113,114)}$ Interestingly, estrogen adaptively influences sensory gating ${ }^{115)}$; i.e., estrogen deficient state can potentially lead to sensory gating deficits. Contextually, it is striking to note that estrogen deficient state is implicated in the pathogenesis of schizophrenia. ${ }^{116,117)}$

The following observations offer robust support to the protective effect of estrogens in schizophrenia (as reviewed in the article of Kulkarni et al. ${ }^{116)}$ ). Women with schizophrenia present with their first episode on average 
about 5 years later than their male counterparts. Life cycle studies have shown that women are more vulnerable for either a first episode or a relapse of existing illness at 2 major periods of hormonal change: during the postpartum period (associated with a decrease in estrogen levels) and during menopause (when estrogen production decreases). Conversely, chronic psychoses and relapse rates improve during pregnancy, when estrogen plasma levels are high. Levels of psychopathological symptoms have also been observed to fluctuate with menstrual cycle phases. Exacerbation or recurrence of psychosis is observed during low estrogen phases of the menstrual cycle, and psychopathological behavior improves during high-estrogen phases. Recently, it has been proposed that estrogen might have beneficial effects in men with schizophrenia too. ${ }^{118)}$ Raloxifene, which is a selective estrogen receptor modulator is considered because of its specific effects on brain; raloxifene has been shown to decrease the IL-6 expression $^{112)}$ and very recently, raloxifene has been shown to have beneficial effects in schizophrenia patients. ${ }^{119)}$

The implication of estrogen in the pathogenesis of schizophrenia becomes more interesting with relevance to the GBG hypothesis especially in the context of recent developments in the domain of developmental programming research studies. ${ }^{120,121)}$ These studies strongly implicate prenatal sex hormonal imbalance to result in persistent postnatal disturbances in the way in which various bodily systems react to the influence of androgens/estrogens as well as the relevant feedback systems. ${ }^{120,121)}$ Earlier studies that suggested sex hormones to modulate lateralization of cognitive function ${ }^{122)}$ have been strengthened by later observations that demonstrated a greater left-hemisphere advantage for a verbal dichotic listening task during the mid-luteal phase, when estrogens and progesterone are at higher level, than during the menstrual period, when the levels of these hormones are very low. On the contrary, during the mid-luteal phase, using an experiment involving musical chord dichotic recognition listening task that is predominantly dependent upon right-hemisphere neural processes, a right-hemisphere advantage was observed. ${ }^{123)}$ Extending these reports, it was reported that estrogen replacement therapy influences cerebral lateralization processes as evaluated using an odor memory/discrimination task $^{124)}$ adding further strength to the proposition that estrogen might 'adaptively' influence cerebral asymmetry aberrations in schizophrenia possibly through concurrent immunomodulation; this needs further systematic evaluation.

\section{CONCLUSIONS AND FUTURE PERSPECTIVES}

In summary, the disparate lines of evidence that have been summarized in this review emphasize the role of immune-inflammatory aberrations and their interactions with cerebral asymmetry abnormalities in contemporary understanding of schizophrenia pathogenesis. However, studies in human subjects that have employed the overarching paradigms that have concurrently evaluated the relationship between immune-inflammatory aberrations and brain asymmetry abnormalities especially in the context of schizophrenia are very few. Such newer paradigms need further systematic research studies to understand the complex interactions of these sex hormone systems and immune factors in the pathogenesis of schizophrenia through putative modulation of cerebral lateralization processes possibly in the prenatal stage of brain development.

\section{Acknowledgments}

This work is supported by the Wellcome Trust / DBT India Alliance Senior Fellowship Research Grant to GV (500236/Z/11/Z).

\section{REFERENCES}

1. van Os J, Kapur S. Schizophrenia. Lancet 2009;374:635645 .

2. Keshavan MS, Tandon R, Boutros NN, Nasrallah HA. Schizophrenia, "just the facts": what we know in 2008 Part 3: neurobiology. Schizophr Res 2008;106:89-107.

3. Rapoport JL, Addington AM, Frangou S, Psych MR. The neurodevelopmental model of schizophrenia: update 2005. Mol Psychiatry 2005;10:434-449.

4. Nicodemus KK, Marenco S, Batten AJ, Vakkalanka R, Egan MF, Straub RE, et al. Serious obstetric complications interact with hypoxia-regulated/vascular-expression genes to influence schizophrenia risk. Mol Psychiatry 2008;13:873-877.

5. Brown AS, Derkits EJ. Prenatal infection and schizophrenia: a review of epidemiologic and translational studies. Am J Psychiatry 2010;167:261-280.

6. Brown AS. Exposure to prenatal infection and risk of schizophrenia. Front Psychiatry 2011;2:63.

7. Madhusudan A, Vogel P, Knuesel I. Impact of prenatal immune system disturbances on brain development. J Neuroimmune Pharmacol 2013;8:79-86.

8. Garay PA, McAllister AK. Novel roles for immune molecules in neural development: implications for neurodevelopmental disorders. Front Synaptic Neurosci 2010;2:136.

9. Geschwind N, Galaburda AM. Cerebral lateralization. Biological mechanisms, associations, and pathology: III. A hypothesis and a program for research. Arch Neurol 1985;42: 634-654.

10. Geschwind N, Galaburda AM. Cerebral lateralization. Biological mechanisms, associations, and pathology: II. A hypothesis and a program for research. Arch Neurol 1985;42: 
521-552.

11. Geschwind N, Galaburda AM. Cerebral lateralization. Biological mechanisms, associations, and pathology: I. A hypothesis and a program for research. Arch Neurol 1985;42: 428-459.

12. Stoyanov Z, Nikolova P, Pashalieva I. Season of birth, Geschwind and Galaburda hypothesis, and handedness. Laterality 2011;16:607-619.

13. Stoyanov Z, Decheva L, Pashalieva I, Nikolova P. Brain asymmetry, immunity, handedness. Cent Eur J Med 2012;7: 1-8.

14. Tochigi M, Okazaki Y, Kato N, Sasaki T. What causes seasonality of birth in schizophrenia? Neurosci Res 2004; 48:1-11.

15. Patterson PH. Neuroscience. Maternal effects on schizophrenia risk. Science 2007;318:576-577.

16. Smith SE, Li J, Garbett $\mathrm{K}$, Mirnics $\mathrm{K}$, Patterson $\mathrm{PH}$. Maternal immune activation alters fetal brain development through interleukin-6. J Neurosci 2007;27:10695-10702.

17. Brown AS. Prenatal infection as a risk factor for schizophrenia. Schizophr Bull 2006;32:200-202.

18. Eyles D, Feldon J, Meyer U. Schizophrenia: do all roads lead to dopamine or is this where they start? Evidence from two epidemiologically informed developmental rodent models. Transl Psychiatry 2012;2:e81.

19. Ashdown H, Dumont Y, Ng M, Poole S, Boksa P, Luheshi GN. The role of cytokines in mediating effects of prenatal infection on the fetus: implications for schizophrenia. Mol Psychiatry 2006;11:47-55.

20. Beloosesky R, Gayle DA, Ross MG. Maternal N-acetylcysteine suppresses fetal inflammatory cytokine responses to maternal lipopolysaccharide. Am J Obstet Gynecol 2006; 195:1053-1057.

21. Meyer U, Schwendener S, Feldon J, Yee BK. Prenatal and postnatal maternal contributions in the infection model of schizophrenia. Exp Brain Res 2006;173:243-257.

22. Dugan LL, Ali SS, Shekhtman G, Roberts AJ, Lucero J, Quick KL, et al. IL-6 mediated degeneration of forebrain GABAergic interneurons and cognitive impairment in aged mice through activation of neuronal NADPH oxidase. PLoS One 2009;4:e5518.

23. Samuelsson AM, Jennische E, Hansson HA, Holmäng A. Prenatal exposure to interleukin-6 results in inflammatory neurodegeneration in hippocampus with $N M D A / G A B A(A)$ dysregulation and impaired spatial learning. Am J Physiol Regul Integr Comp Physiol 2006;290:R1345-R1356.

24. Carpenter LL, Gawuga CE, Tyrka AR, Lee JK, Anderson GM, Price LH. Association between plasma IL-6 response to acute stress and early-life adversity in healthy adults. Neuropsychopharmacology 2010;35:2617-2623.

25. Sasayama D, Hattori K, Wakabayashi C, Teraishi T, Hori $\mathrm{H}$, Ota $\mathrm{M}$, et al. Increased cerebrospinal fluid interleukin-6 levels in patients with schizophrenia and those with major depressive disorder. J Psychiatr Res 2013;47:401-406.

26. Potvin S, Stip E, Sepehry AA, Gendron A, Bah R, Kouassi E. Inflammatory cytokine alterations in schizophrenia: a systematic quantitative review. Biol Psychiatry 2008;63:801808.

27. Paul-Samojedny M, Kowalczyk M, Suchanek R, Owczarek A, Fila-Danilow A, Szczygiel A, et al. Functional polymorphism in the interleukin-6 and interleukin-10 genes in patients with paranoid schizophrenia-a case-control study. $J$ Mol Neurosci 2010;42:112-119.

28. Marsland AL, Gianaros PJ, Abramowitch SM, Manuck SB, Hariri AR. Interleukin-6 covaries inversely with hippo- campal grey matter volume in middle-aged adults. Biol Psychiatry 2008;64:484-490.

29. Behrens MM, Ali SS, Dugan LL. Interleukin-6 mediates the increase in NADPH-oxidase in the ketamine model of schizophrenia. J Neurosci 2008;28:13957-13966.

30. Bauer S, Kerr BJ, Patterson PH. The neuropoietic cytokine family in development, plasticity, disease and injury. Nat Rev Neurosci 2007;8:221-232.

31. Sargent IL, Borzychowski AM, Redman CW. Immunoregulation in normal pregnancy and pre-eclampsia: an overview. Reprod Biomed Online 2006;13:680-686.

32. Sargent IL, Borzychowski AM, Redman CW. NK cells and human pregnancy--an inflammatory view. Trends Immunol 2006;27:399-404.

33. Zimmerman AW, Connors SL, Matteson KJ, Lee LC, Singer HS, Castaneda JA, et al. Maternal antibrain antibodies in autism. Brain Behav Immun 2007;21:351-357.

34. Sparkman NL, Buchanan JB, Heyen JR, Chen J, Beverly JL, Johnson RW. Interleukin-6 facilitates lipopolysaccharide-induced disruption in working memory and expression of other proinflammatory cytokines in hippocampal neuronal cell layers. J Neurosci 2006;26:10709-10716.

35. Murphy PG, Borthwick LA, Altares M, Gauldie J, Kaplan D, Richardson PM. Reciprocal actions of interleukin-6 and brain-derived neurotrophic factor on rat and mouse primary sensory neurons. Eur J Neurosci 2000;12:1891-1899.

36. Buckley PF, Pillai A, Howell KR. Brain-derived neurotrophic factor: findings in schizophrenia. Curr Opin Psychiatry 2011;24:122-127.

37. Favalli G, Li J, Belmonte-de-Abreu P, Wong AH, Daskalakis ZJ. The role of BDNF in the pathophysiology and treatment of schizophrenia. J Psychiatr Res 2012;46:1-11.

38. Pezawas L, Verchinski BA, Mattay VS, Callicott JH, Kolachana BS, Straub RE, et al. The brain-derived neurotrophic factor val66met polymorphism and variation in human cortical morphology. J Neurosci 2004;24:10099-10102.

39. Shoval G, Weizman A. The possible role of neurotrophins in the pathogenesis and therapy of schizophrenia. Eur Neuropsychopharmacol 2005;15:319-329.

40. Meyer U, Feldon J, Yee BK. A review of the fetal brain cytokine imbalance hypothesis of schizophrenia. Schizophr Bull 2009;35:959-972.

41. Venkatasubramanian G, Chittiprol S, Neelakantachar N, Naveen MN, Thirthall J, Gangadhar BN, et al. Insulin and insulin-like growth factor-1 abnormalities in antipsychoticnaive schizophrenia. Am J Psychiatry 2007;164:1557-1560.

42. Doré S, Kar S, Quirion R. Insulin-like growth factor I protects and rescues hippocampal neurons against betaamyloid- and human amylin-induced toxicity. Proc Natl Acad Sci U S A 1997;94:4772-4777.

43. de Martino M, Galli L, Chiarelli F, Verrotti A, Rossi ME, Bindi G, et al. Interleukin- 6 release by cultured peripheral blood mononuclear cells inversely correlates with height velocity, bone age, insulin-like growth factor-I, and insulinlike growth factor binding protein-3 serum levels in children with perinatal HIV-1 infection. Clin Immunol 2000;94:212218.

44. Hsiao EY, Patterson PH. Activation of the maternal immune system induces endocrine changes in the placenta via IL-6. Brain Behav Immun 2011;25:604-615.

45. Harrison PJ. The hippocampus in schizophrenia: a review of the neuropathological evidence and its pathophysiological implications. Psychopharmacology (Berl) 2004;174:151-162.

46. Heckers S. The hippocampus in schizophrenia. Am J Psychiatry 2004;161:2138-2139. 
47. Tamminga $\mathrm{CA}$, Stan $\mathrm{AD}$, Wagner $\mathrm{AD}$. The hippocampal formation in schizophrenia. Am J Psychiatry 2010;167:11781193.

48. Lee E, Son H. Adult hippocampal neurogenesis and related neurotrophic factors. BMB Rep 2009;42:239-244.

49. Fishman D, Faulds G, Jeffery R, Mohamed-Ali V, Yudkin JS, Humphries S, et al. The effect of novel polymorphisms in the interleukin-6 (IL-6) gene on IL-6 transcription and plasma IL-6 levels, and an association with systemic-onset juvenile chronic arthritis. J Clin Invest 1998;102:1369-1376.

50. Broca P. Rapport sur un memoire de M. Armand de Fleury intitulé: De l'inegalité dynamique des deux hemisphères cerébraux. Bull Acad Med 1877;6:508-539.

51. Crow TJ. Cerebral asymmetry and the lateralization of language: core deficits in schizophrenia as pointers to the gene. Curr Opin Psychiatry 2004;17:97-106.

52. Cantalupo C, Hopkins WD. Asymmetric Broca's area in great apes. Nature 2001;414:505.

53. Gannon PJ, Holloway RL, Broadfield DC, Braun AR. Asymmetry of chimpanzee planum temporale: humanlike pattern of Wernicke's brain language area homolog. Science 1998; 279:220-222.

54. Glasel H, Leroy F, Dubois J, Hertz-Pannier L, Mangin JF, Dehaene-Lambertz G. A robust cerebral asymmetry in the infant brain: the rightward superior temporal sulcus. Neuroimage 2011;58:716-723.

55. Buxhoeveden DP, Switala AE, Litaker M, Roy E, Casanova MF. Lateralization of minicolumns in human planum temporale is absent in nonhuman primate cortex. Brain Behav Evol 2001;57:349-358.

56. Moore JK, Guan YL. Cytoarchitectural and axonal maturation in human auditory cortex. J Assoc Res Otolaryngol 2001;2:297-311.

57. Chance SA, Casanova MF, Switala AE, Crow TJ, Esiri MM. Minicolumn thinning in temporal lobe association cortex but not primary auditory cortex in normal human ageing. Acta Neuropathol 2006;111:459-464.

58. Shenton ME, Dickey CC, Frumin M, McCarley RW. A review of MRI findings in schizophrenia. Schizophr Res 2001;49:1-52.

59. Chance SA, Casanova MF, Switala AE, Crow TJ. Auditory cortex asymmetry, altered minicolumn spacing and absence of ageing effects in schizophrenia. Brain 2008;131:31783192.

60. Satz P, Green MF. Atypical handedness in schizophrenia: some methodological and theoretical issues. Schizophr Bull 1999;25:63-78

61. Sommer I, Ramsey N, Kahn R, Aleman A, Bouma A. Handedness, language lateralisation and anatomical asymmetry in schizophrenia: meta-analysis. Br J Psychiatry 2001;178: 344-351.

62. Dragovic M, Hammond G. Handedness in schizophrenia: a quantitative review of evidence. Acta Psychiatr Scand 2005. 111:410-419.

63. Crow TJ. Is schizophrenia the price that Homo sapiens pays for language? Schizophr Res 1997;28:127-141.

64. Crow TJ. The 'big bang' theory of the origin of psychosis and the faculty of language. Schizophr Res 2008;102:31-52.

65. Oertel V, Knöchel C, Rotarska-Jagiela A, Schönmeyer R, Lindner $\mathrm{M}$, van de Ven $\mathrm{V}$, et al. Reduced laterality as a trait marker of schizophrenia-evidence from structural and functional neuroimaging. J Neurosci 2010;30:2289-2299.

66. Geschwind N, Galaburda AM. Cerebral lateralization: Biological mechanisms, associations, and pathology. Cambridge: MIT Press; 1987.
67. Geschwind N, Behan P. Left-handedness: association with immune disease, migraine, and developmental learning disorder. Proc Natl Acad Sci U S A 1982;79:5097-5100.

68. Chengappa KN, Cochran J, Rabin BS, Ganguli R. Handedness and autoantibodies. Lancet 1991;338:694.

69. Chengappa KN, Ganguli R, Ulrich R, Rabin BS, Cochran $\mathrm{J}$, Brar JS, et al. The prevalence of autoantibodies among right and left handed schizophrenic patients and control subjects. Biol Psychiatry 1992;32:803-811.

70. Chengappa KN, Ganguli R, Yang ZW, Brar JS, Li L, Rabin BS. Left-handed first-episode, neuroleptic-naive schizophrenic patients have a higher prevalence of autoantibodies. Schizophr Res 1992;8:75-80.

71. Hassler M. Anomalous dominance, immune parameters, and spatial ability. Int J Neurosci 1993;68:145-156.

72. Hassler M, Gupta D. Functional brain organization, handedness, and immune vulnerability in musicians and non-musicians. Neuropsychologia 1993;31:655-660.

73. Morfit NS, Weekes NY. Handedness and immune function. Brain Cogn 2001;46:209-213.

74. Pennington BF, Smith SD, Kimberling WJ, Green PA, Haith MM. Left-handedness and immune disorders in familial dyslexics. Arch Neurol 1987;44:634-639.

75. Searleman A, Fugagli AK. Suspected autoimmune disorders and left-handedness: evidence from individuals with diabetes. Crohn's disease and ulcerative colitis. Neuropsychologia 1987;25:367-374.

76. Smith BD, Meyers MB, Kline R. For better or for worse: left-handedness, pathology, and talent. J Clin Exp Neuropsychol 1989;11:944-958.

77. McManus IC, Bryden MP. Geschwind's theory of cerebral lateralization: developing a formal, causal model. Psychol Bull 1991;110:237-253.

78. Berenbaum SA, Denburg SD. Evaluating the empirical support for the role of testosterone in the Geschwind-BehanGalaburda model of cerebral lateralization: commentary on Bryden, McManus, and Bulman-Fleming. Brain Cogn 1995; 27:79-83.

79. Pennell LM, Galligan CL, Fish EN. Sex affects immunity. J Autoimmun 2012;38:J282-J291.

80. Peeva E, Zouali M. Spotlight on the role of hormonal factors in the emergence of autoreactive B-lymphocytes. Immunol Lett 2005;101:123-143.

81. Kang DH, Davidson RJ, Coe CL, Wheeler RE, Tomarken AJ, Ershler WB. Frontal brain asymmetry and immune function. Behav Neurosci 1991;105:860-869.

82. Neveu PJ. Asymmetrical brain modulation of the immune response. Brain Res Brain Res Rev 1992;17:101-107.

83. Lengen $C$, Regard $M$, Joller $H$, Landis $T$, Lalive $P$. Anomalous brain dominance and the immune system: do left-handers have specific immunological patterns? Brain Cogn 2009;69:188-193.

84. Müller W, Groothuis TG, Kasprzik A, Dijkstra C, Alatalo $\mathrm{RV}$, Siitari H. Prenatal androgen exposure modulates cellular and humoral immune function of black-headed gull chicks. Proc Biol Sci 2005;272:1971-1977.

85. Debnath M, Cannon DM, Venkatasubramanian G. Variation in the major histocompatibility complex [MHC] gene family in schizophrenia: associations and functional implications. Prog Neuropsychopharmacol Biol Psychiatry 2013;42:4962.

86. Wierzba-Bobrowicz T, Lewandowska E, Kosno-Kruszewska E, Lechowicz W, Pasennik E, Schmidt-Sidor B. Degeneration of microglial cells in frontal and temporal lobes of chronic schizophrenics. Folia Neuropathol 2004;42:157-165. 
87. Steiner J, Mawrin C, Ziegeler A, Bielau H, Ullrich O, Bernstein HG, et al. Distribution of HLA-DR-positive microglia in schizophrenia reflects impaired cerebral lateralization. Acta Neuropathol 2006;112:305-316.

88. Billiards SS, Haynes RL, Folkerth RD, Trachtenberg FL, Liu $\mathrm{LG}$, Volpe JJ, et al. Development of microglia in the cerebral white matter of the human fetus and infant. J Comp Neurol 2006;497:199-208.

89. Barres BA. The mystery and magic of glia: a perspective on their roles in health and disease. Neuron 2008;60:430440.

90. Giaume C, Kirchhoff F, Matute C, Reichenbach A, Verkhratsky A. Glia: the fulcrum of brain diseases. Cell Death Differ 2007;14:1324-1335.

91. Bilbo SD, Schwarz JM. Early-life programming of later-life brain and behavior: a critical role for the immune system. Front Behav Neurosci 2009;3:14.

92. Monji A, Kato T, Kanba S. Cytokines and schizophrenia: Microglia hypothesis of schizophrenia. Psychiatry Clin Neurosci 2009;63:257-265.

93. Miller BJ, Buckley P, Seabolt W, Mellor A, Kirkpatrick B. Meta-analysis of cytokine alterations in schizophrenia: clinical status and antipsychotic effects. Biol Psychiatry 2011;70:663-671.

94. Doorduin J, de Vries EF, Willemsen AT, de Groot JC, Dierckx RA, Klein HC. Neuroinflammation in schizophrenia-related psychosis: a PET study. J Nucl Med 2009; 50:1801-1807.

95. Fujita Y, Ishima T, Kunitachi S, Hagiwara H, Zhang L, Iyo $\mathrm{M}$, et al. Phencyclidine-induced cognitive deficits in mice are improved by subsequent subchronic administration of the antibiotic drug minocycline. Prog Neuropsychopharmacol Biol Psychiatry 2008;32:336-339.

96. Hashimoto K. Microglial activation in schizophrenia and minocycline treatment. Prog Neuropsychopharmacol Biol Psychiatry 2008;32:1758-1759; author reply 1760.

97. Hashimoto K. Abnormality of cerebral perfusion in the posterior cingulate gyrus of a refractory patient with schizophrenia and minocycline treatment. Prog Neuropsychopharmacol Biol Psychiatry 2010;34:1132; author reply 1133-1134.

98. Chaudhry IB, Hallak J, Husain N, Minhas F, Stirling J, Richardson $\mathrm{P}$, et al. Minocycline benefits negative symptoms in early schizophrenia: a randomised double-blind placebocontrolled clinical trial in patients on standard treatment. $J$ Psychopharmacol 2012;26:1185-1193.

99. Levkovitz Y, Mendlovich S, Riwkes S, Braw Y, LevkovitchVerbin $\mathrm{H}, \mathrm{Gal} \mathrm{G}$, et al. A double-blind, randomized study of minocycline for the treatment of negative and cognitive symptoms in early-phase schizophrenia. J Clin Psychiatry 2010;71:138-149.

100. Jhamnani K, Shivakumar V, Kalmady S, Rao NP, Venkatasubramanian G. Successful use of add-on minocycline for treatment of persistent negative symptoms in schizophrenia. J Neuropsychiatry Clin Neurosci 2013;25:E06-E07.

101. Romero E, Guaza C, Castellano B, Borrell J. Ontogeny of sensorimotor gating and immune impairment induced by prenatal immune challenge in rats: implications for the etiopathology of schizophrenia. Mol Psychiatry 2010;15: 372-383.

102. Borrell J, Vela JM, Arévalo-Martin A, Molina-Holgado E, Guaza C. Prenatal immune challenge disrupts sensorimotor gating in adult rats. Implications for the etiopathogenesis of schizophrenia. Neuropsychopharmacology 2002;26:204-215.

103. Mcghie A, Chapman J. Disorders of attention and percep- tion in early schizophrenia. Br J Med Psychol 1961;34: 103-116.

104. Venables PH. Selectivity of attention, withdrawal, and cortical activation. Studies in chronic schizophrenia. Arch Gen Psychiatry 1963;9:74-78.

105. Williams TJ, Nuechterlein KH, Subotnik KL, Yee CM. Distinct neural generators of sensory gating in schizophrenia. Psychophysiology 2011;48:470-478.

106. Patterson JV, Hetrick WP, Boutros NN, Jin Y, Sandman C, Stern $\mathrm{H}$, et al. P50 sensory gating ratios in schizophrenics and controls: a review and data analysis. Psychiatry Res 2008; 158:226-247.

107. Potter D, Summerfelt A, Gold J, Buchanan RW. Review of clinical correlates of P50 sensory gating abnormalities in patients with schizophrenia. Schizophr Bull 2006;32: 692-700.

108. Kim SH, Lee JM, Kim HP, Jang DP, Shin YW, Ha TH, et al. Asymmetry analysis of deformable hippocampal model using the principal component in schizophrenia. Hum Brain Mapp 2005;25:361-369.

109. Qiu A, Wang L, Younes L, Harms MP, Ratnanather JT, Miller MI, et al. Neuroanatomical asymmetry patterns in individuals with schizophrenia and their non-psychotic siblings. Neuroimage 2009;47:1221-1229.

110. Lang TJ. Estrogen as an immunomodulator. Clin Immunol 2004;113:224-230.

111. Kalaitzidis D, Gilmore TD. Transcription factor cross-talk: the estrogen receptor and NF-kappaB. Trends Endocrinol Metab 2005;16:46-52.

112. Paimela T, Hyttinen JM, Viiri J, Ryhänen T, Karvonen $\mathrm{MK}$, Unkila $\mathrm{M}$, et al. Influence of selective estrogen receptor modulators on interleukin-6 expression in human retinal pigment epithelial cells (ARPE-19). Eur J Pharmacol 2010;640:219-225.

113. Gingerich S, Kim GL, Chalmers JA, Koletar MM, Wang $\mathrm{X}$, Wang $\mathrm{Y}$, et al. Estrogen receptor $a$ and G-protein coupled receptor 30 mediate the neuroprotective effects of $17 \beta$-estradiol in novel murine hippocampal cell models. Neuroscience 2010;170:54-66.

114. Brann DW, Dhandapani K, Wakade C, Mahesh VB, Khan MM. Neurotrophic and neuroprotective actions of estrogen: basic mechanisms and clinical implications. Steroids 2007;72:381-405.

115. Meng JL, Ma YY, Luo HY, Kong SZ, He YW, Dong BC, et al. Estrogen protects the dopaminergic neurons in substantia nigra against damage induced by 6-hydroxydopamine. Sheng Li Xue Bao 2008;60:369-374.

116. Kulkarni J, de Castella A, Fitzgerald PB, Gurvich CT, Bailey M, Bartholomeusz C, et al. Estrogen in severe mental illness: a potential new treatment approach. Arch Gen Psychiatry 2008;65:955-960.

117. Kulkarni J, Riedel A, de Castella AR, Fitzgerald PB, Rolfe TJ, Taffe J, et al. Estrogen - a potential treatment for schizophrenia. Schizophr Res 2001;48:137-144.

118. Kulkarni J, de Castella A, Headey B, Marston N, Sinclair $\mathrm{K}$, Lee $\mathrm{S}$, et al. Estrogens and men with schizophrenia: is there a case for adjunctive therapy? Schizophr Res 2011;125:278-283.

119. Kulkarni J, Gurvich C, Lee SJ, Gilbert H, Gavrilidis E, de Castella A, et al. Piloting the effective therapeutic dose of adjunctive selective estrogen receptor modulator treatment in postmenopausal women with schizophrenia. Psychoneuroendocrinology 2010;35:1142-1147.

120. Salvetti NR, Ortega HH, Veiga-Lopez A, Padmanabhan V. Developmental programming: impact of prenatal testo- 
sterone excess on ovarian cell proliferation and apoptotic factors in sheep. Biol Reprod 2012;87:22, 1-10.

121. Abi Salloum B, Herkimer C, Lee JS, Veiga-Lopez A, Padmanabhan V. Developmental programming: prenatal and postnatal contribution of androgens and insulin in the reprogramming of estradiol positive feedback disruptions in prenatal testosterone-treated sheep. Endocrinology 2012; 153:2813-2822.

122. Kimura D. Sex, sexual orientation and sex hormones in- fluence human cognitive function. Curr Opin Neurobiol 1996;6:259-263.

123. Sanders G, Wenmoth D. Verbal and music dichotic listening tasks reveal variations in functional cerebral asymmetry across the menstrual cycle that are phase and task dependent. Neuropsychologia 1998;36:869-874.

124. Doty RL, Kisat M, Tourbier I. Estrogen replacement therapy induces functional asymmetry on an odor memory/ discrimination test. Brain Res 2008;1214:35-39. 